\title{
Co-regulation role of endogenous hormones and transcriptomics profiling under cold stress in Tetrastigma hemsleyanum
}

\section{Xin Peng ( $\square$ px4142@163.com)}

Zhejiang pharmaceutical college

Wenyu Qiu

Zhejiang Pharmaceutical College

\section{Mingjie Li}

Fujian Agriculture and Forestry University

\section{Hao Wu}

Zhejiang Pharmaceutical College

\section{Hongjiang Chen}

Zhejiang pharmaceutical college

\section{Zhongyi Zhang}

Fujian Agriculture and Forestry University

\section{Research article}

Keywords: Tetrastigma hemsleyanum, Transcriptome, Cold stress, Phytohormone

Posted Date: March 3rd, 2020

DOI: https://doi.org/10.21203/rs.3.rs-15749/v1

License: () (1) This work is licensed under a Creative Commons Attribution 4.0 International License. Read Full License

Version of Record: A version of this preprint was published at Journal of Plant Growth Regulation on October 26th, 2020. See the published version at https://doi.org/10.1007/s00344-020-10246-6. 


\section{Abstract}

Background: Tetrastigma hemsleyanum Diels et Gilg is a valuable medicinal herb, Chilling sensitivity is the dominant environmental factor limiting the artificial domestication of the plant. Hormone-related gene regulation and hormone signaling pathways in response to cold stress in T. hemsleyanum remain unknown.

Results: Some key genes involved in hormones biosynthesis, such as ZEP and NCED genes of ABA biosynthesis, GA2ox, GA3ox, and GA20ox genes of GA biosynthesis, ACO genes of ET biosynthesis pathway were screened to be crucial in cold response. Consistently, the response of $A B A$ and $A B A / G A 1+3$ to cold stress was prior to that of GA1+3, ZR, ABA/IAA, and ABA/ZR. The increasing changes in ABA/GA1+3 turned to a steep decline with the extension of stress time, which might be one factor contributing to cold-sensitivity of T. hemsleyanum under prolonged stress. The cold tolerance of T. hemsleyanum would be enhanced by ABA but repressed by GA3 when each phytohormone was applied alone. The ABA-mediated promotion and GA-mediated repression of cold tolerance could both be attenuated by the co-application of the two phytohormones within $6 \mathrm{~h}$. When the biosynthesis of endogenous $A B A$ and GA were inhibited by FLU and PAC, respectively, the effects of GA and ABA treatment were reversed partially.

Conclusions: In summary, we presented the first study of global expression patterns of hormone-regulated transcripts in T. hemsleyanum. This study suggested that GA and ABA could work antagonistically to balance the responses to cold in T. hemsleyanum. PAC, a GA biogenesis inhibitor, as well as exogenous ABA, might be potential plant growth regulators that can promote cold tolerance of T. hemsleyanum. The study also provided valuable hints in revealing a new theoretical basis and potential candidate genes that govern cold tolerance of T. hemsleyanum.

\section{Background}

Tetrastigma hemsleyanum Diels et Gilg is a perennial herb, and its underground root contains abundant flavonoids and polysaccharides that are beneficial to human health [1, 2]. Due to over exploitation, wild resources are on the verge of extinction in recent years. However, it is considered to be a cold-sensitive species, with the best growing temperatures ranging from $20^{\circ} \mathrm{C}$ to $30^{\circ} \mathrm{C}$. Low temperature susceptibility has become the dominant environmental factor limiting the artificial domestication of T. hemsleyanum. So the development of cold stress tolerance in T. hemsleyanum is important for improving production but it is hindered by lack of more precise physiological knowledge. A better understanding of the gene expression profile of T. hemsleyanum under cold stress is imperative, which could be an important theoretical basis to study cold tolerance mechanisms for improving the cold resistance.

Studies have been reported to provide evidence of global changes in gene expression in response to cold stress [3, 4], which highlighted the significance of transcriptional regulation in plant adaptation to low temperature.

Phytohormone are generally considered to participate in the regulation of organ differentiation and development. Recently, the development of stress tolerance has been reported in some plants to be accompanied by changes in hormonal-related genes expression and subsequently physiochemical modifications [5]. Plants could survive from stress by utilizing hormones to regulate and coordinate growth. These hormones-related genes could act in the upstream of stress response and trigger hormone signal pathway. 
Abscisic acid ( $A B A$ ) has been shown to mediate plant responses to various kinds of environmental stresses like cold [6], drought [7], and so on. Exogenous ABA application before cold stress improved resistance of sugarcane seedlings by increasing contents of proline and soluble sugar [8]. Recent studies showed the existence of potential co-regulation among the ABA and other hormones in plants. For example, auxin controlled seed dormancy by activating the $A B A$ response [9]. Changes in $A B A$ status can regulate cytokinin (CK) concentrations via altering cytokinin oxidase activity [10]. Moreover, the balance between ABA and gibberellic acid (GA) has been proposed to be correlated with stress responses [11]. Both the elevated content of endogenous ABA and the higher proportion of $A B A / I A A$ and $A B A / G A$ played an important role in kiwifruit resistance to cold [12].

However, hormone-related gene regulation and hormone signaling pathways in response to cold stress in $\mathrm{T}$. hemsleyanum remain unknown. Our aim was therefore to elucidate the effects, as well as the transcriptomic adaptive mechanisms of cold stress on endogenous plant hormones in T. hemsleyanum. The results will facilitate the identification of potential candidate genes involved in cold stress tolerance in T. hemsleyanum, which should contribute to improving cold tolerance in future breeding programs, and should contribute to our understanding of the hormone responsive regulatory mechanisms of plant in response to environmental stress.

\section{Results}

In previous research, we measured the phenotypes and some physiological and biochemical parameters [13]. Our results showed that the $0{ }^{\circ} \mathrm{C}$ exposure was the optimum treatment temperature. The physiological and biochemical parameters remained essentially stable during $0 \mathrm{~h}$ and $8 \mathrm{~h}$, significantly changed from $12 \mathrm{~h}$ to $48 \mathrm{~h}$, and remained stable after $48 \mathrm{~h}$. So RNA-seq was employed to investigate the changes in gene expression under $0{ }^{\circ} \mathrm{C}$ exposure for four time points $(0,12,24$, and $48 \mathrm{~h})$.

\section{Overview Of Transcriptome Sequencing}

Four T. hemsleyanum leaves cDNA libraries were constructed and sequenced. A total of 216,043,064 raw reads were generated. After removing low-quality reads and adapter sequences, 181,412,182 clean reads were obtained, with $45,346,334 ; 46,460,402 ; 44,804,246 ; 44,801,200$ clean reads generated for the $0,12,24$, and 48 h treatment group libraries, respectively.

We obtained 106,275 unigenes annotated in Nr, NT, BLASTX, and BLASTP databases with an average length of 676 bp and N50 of 1121 bp (Fig. 1). Among these unigenes, 18082 unigenes had hits in all four public databases with informative annotations. 53,511 unigenes had hits that significantly matched against the Nt database (50.35\% of all unigenes). According to Nt annotations, 41,743 unigenes were matched to Vitis vinifera, followed by Zea mays. The E-value distribution of the $\mathrm{Nr}$ blast results indicated that $81.91 \%$ of the matched sequences $(43,831)$ had strong homologies (smaller than $1.0 \mathrm{e}^{-60}$ ), while the other $18.09 \%$ had homologies that ranged between $1.0 \mathrm{e}^{-5}$ to $1.0 \mathrm{e}^{-60}$. To produce more definitive annotations, the Nr, BLASTX, and BLASTP database were also used to align all of the unigenes. 43,$827 ; 28,775$ and 19,598 high-score annotate unigenes were matched, respectively.

\section{Go Enrichment Analysis Of Degs}

To characterize the functional classifications of differentially expressed genes, GO and KEGG analyses were performed to access the distributions of functional categories. In the 12,24 , and 48 h cold treatment samples, 
8205(72.4\%), 14400(83.5\%), and 15402(85.1\%) unigenes were annotated in GO, respectively. In the $12 \mathrm{~h}$ treatment group (Fig. 2A), 'membrane' (1194; 77.5\% of 1541), 'membrane part' (986; 63.4\% of 1541), and intrinsic component of membrane' (943; $63.4 \%$ of 1541$)$ were the terms that dominated in the cellular component category. Response to stimulus(972; $48.1 \%$ of 2022) 'response to stress(652; 32.3\% of 2022)' 'defense response(329; $16.3 \%$ of 2022 )' 'hormone-mediated signaling (164; 8.11\% of 2022)' were the most representative terms in the biological process category. 'oxidoreductase activity' (397; $25.88 \%$ of 1534), 'nucleic acid binding transcription factor activity' (247; $16.0 \%$ of 1534$)$ and 'sequence-specific DNA transcription factor binding transcription factor activity' $(247 ; 16.0 \%$ of 1534) were the most abundant terms in the molecular function category. During the $24 \mathrm{~h}$ (Fig. $2 \mathrm{~B}$ ) and $48 \mathrm{~h}$ (Fig. 2C)cold stress treatment, 'response to stimulus', 'response to stress' remained relatively stable in the biological process category, while 'response to hormone' and 'response to endogenous stimulus' skyrocketed in $24 \mathrm{~h}$ cold treatment samples. 'membrane', 'membrane part', 'intrinsic component of membrane' remained relatively stable in the cellular component category, while 'integral component of membrane' was suddenly increased in $24 \mathrm{~h}$ cold treatment samples. 'oxidoreductase activity' remained predominating during the whole cold stress. In the $12 \mathrm{~h}$ cold treatment samples, various of transcription factor activity were the most abundant terms in the molecular function category, such as 'nucleic acid binding transcription factor activity', 'sequence-specific DNA transcription factor binding transcription factor activity'. But with the increase of cold stress time, 'kinsae activity' and 'catalytic activity' dramatically predominated in the molecular function category.

In general, 'response to stimulus', 'response to stress', 'membrane', 'membrane part', 'intrinsic component of membrane', 'oxidoreductase activity' remained predominating during the whole cold stress, while some biological process categories related to endogenous hormone response skyrocketed in the early stress stage, such as 'hormone-mediated signaling' 'response to hormone' and 'response to endogenous stimulus' in $12 \mathrm{~h}$ and $24 \mathrm{~h}$. 'single organism process, 'integral component of membrane', and 'kinsae activity' were suddenly increased in the intermediate stress stage, 'catalytic activity' dramatically predominated in the late stress stage.

\section{Kegg Pathways Enrichment Analysis Of Degs}

Out of total annotated unigenes, 12,936 unigenes were significantly matched to KEGG database and were assigned to 272 KEGG pathways. Pathways displaying significant changes (Q value $\leq 0.05$ ) in response to the cold treatment were identified in each comparison group. The numbers of up-regulated genes and down-regulated genes in enriched pathways for each comparison group were listed in Fig. 3. In the 12 h (Fig. 3A), 24 h (Fig. 3B), and $48 \mathrm{~h}$ (Fig. 3C) cold treatment samples, 12, 6, and 11 KEGG pathways were significantly enriched, respectively. The top 5 enriched pathways of the $12 \mathrm{~h}$ cold treatment sample were 'metabolic pathways', 'biosynthesis of secondary metabolites', 'starch and sucrose metabolism', 'phenylpropanoid biosynthesis', and 'phenylalanine metabolism'. The top 5 enriched pathways of the $24 \mathrm{~h}$ cold treatment sample were 'metabolic pathways', 'biosynthesis of secondary metabolites', 'phenylpropanoid biosynthesis', 'steroid biosynthesis', and 'diterpenoid biosynthesis'. In the $48 \mathrm{~h}$ cold treatment sample, 'metabolic pathways', 'biosynthesis of secondary metabolites', 'phenylpropanoid biosynthesis' were also the top three enriched pathways, but it is noteworthy that various degradation metabolisms started to appear in enriched pathways, such as 'bisphenol degradation', 'polycyclic aromatic hydrocarbon degradation', 'limonene and pinene degradation'.

In general, 'metabolic pathways', 'biosynthesis of secondary metabolites', and 'phenylpropanoid biosynthesis' were common regulated during the whole cold stress stage. The cold responses were characterized by protective response through stimulating secondary metabolism and inhibiting primary metabolism to modulate cellular 
metabolic homeostasis. In the early stress stage, primary metabolism still remained vigorous, such as 'starch and glucose metabolism' and 'phenylalanine metabolism', while the related secondary metabolism enzyme activities were gradually activated. It would lead to the stimulation of secondary metabolisms, such as 'steroid biosynthesis', 'diterpenoid biosynthesis' and so on. With the increase of cold stress time and chilling injury, many degradation pathways were strengthened, such as 'bisphenol degradation', 'polycyclic aromatic hydrocarbon degradation', 'limonene and pinene degradation', and ultimately led to cell apoptosis and necrosis.

\section{Responses Of Hormone-related Genes}

Based on the above overview of gene ontology enrichment and KEGG pathways present during cold treatment, we speculated that endogenous hormone-mediated signaling process would play an important role in early-time responses of T. hemsleyanum under cold exposure. So here we mainly focused on groups of differentially expressed genes involved in different plant hormone biosynthesis pathways, such as Auxin, abscisic acid (ABA), jasmonic acid (JA), gibberellic acid (GA), brassinosteroid (BR), cytokinin (CK) and ethylene (ET) pathways. Some representative hormone-related genes that were differentially expressed during the cold treatment are shown in Supplementary file 1-7. The heat map of hormones-related transcripts of T. hemsleyanum during 0 to $48 \mathrm{~h}$ of cold stress are shown in Fig. 4.

Abscisic acid ( $A B A)$ is an important phytohormone in plant stress response. Its metabolic changes are one of the determinants of endogenous ABA levels and control diverse stress response. Among the mapped enzymes, we identified four 9-cis-epoxycarotenoid dioxygenase (NCED) homologs of the ABA biosynthesis pathway in annotated transcriptome unigene dataset, and all of them were highly expressed with RPKM values $>100$. Two of them were significantly up-regulated by $2.0-2.5$ folds at $24 \mathrm{~h}$. Zeaxanthin epoxidase (ZEP) is another key enzyme in ABA biosynthesis. Three highly expressed ZEP genes were identified, with c95330_g1 and c81504_g1 being the most abundantly and the remarkable differentially expressed. c35330_g2 was the most particularly noteworthy ZEP gene, which was almost undetectable in control and was significantly up-regulated by 9.8 folds and 21 folds under $24 \mathrm{~h}$ and $48 \mathrm{~h}$ of cold treatment, with an increased RPKM value from 0.024 to 0.237 and 0.511 , respectively.

Four indole-3- acetaldehyde oxidase(AA01) genes, two cellulose synthase A catalytic(CESA)genes and three abscisic-aldehyde oxidase(AAO3) genes were all down-regulated significantly under cold stress. So in general, in the ABA biosynthesis pathway, most of ZEP genes were strongly induced significantly during early cold stress, most of NCED genes were up-regulated significantly during the medium stress stage, while other genes were downregulated significantly. But with the increase of cold stress time and chilling injury, all the above genes were downregulated significantly at $48 \mathrm{~h}$.

Four Gibberellin 20 oxidase(GA20ox) genes were identified in our dataset, and the fact of c98675_g1and c93517_g2 were the most highly expressed suggested they may be crucial for gibberellic acid (GA) biosynthesis, with a decreased RPKM value from 214 to 21 and from 430 to 20 under cold stress, respectively. Six Gibberellin 2beta-dioxygenase(GA2ox) genes were identified in our dataset. c92581_g1 was the most highly expressed GA2ox gene, with an increased RPKM value from 1051 to 3426 under cold stress. c93844_g1 was the most remarkable differentially expressed GA2ox gene, with a 7.6 folds significant up-regulation under cold stress, the RPKM value increased from 0.339 to 2.586 . Four GA3ox were identified, among which c78816_g1 was the most highly expressed, with a 6.2 folds significant up-regulation under cold stress. 
All differentially expressed genes in the auxin biosynthesis pathway were down-regulated, including three indole-3acetaldehyde oxidase (AA01)genes, four indole-3-pyruvate monooxygenase (YUCCA) genes, and three abscisicaldehyde oxidase (AAO3) genes.

Five CK riboside 5'-monophosphate phosphoribohydrolase (CRMP_LOG) genes were differentially expressed in cytokinin (CK) biosynthesis pathway. Among them, LOG1, 5, 7 were up-regulated, while LOG3, 8 were downregulated significantly. and the fact of c72906_g1 was the most abundantly and the most remarkable differentially expressed suggested it might be crucial for CK biosynthesis, with an increased RPKM value from 624 to 2292 after $24 \mathrm{~h}$ of cold treatment.

Eight 1-aminocyclopropane-1-carboxylate oxidase(ACO) genes of ethylene (ET) biosynthesis pathway were differentially expressed under cold treatment. Most of them were up-regulated in early stress stage and then maintained a high level, among which c90679_g3 was the most highly and the most remarkably up-regulated ACO gene, with an increased RPKM value from 12152 to 68974 after $24 \mathrm{~h}$ of cold treatment. However, three 1aminocyclopropane-1-carboxylate synthase (ACS) genes of ET biosynthesis pathway were identified to be differentially expressed during cold acclimation. Two of them were down-regulated significantly, with a decreased RPKM value from 112 to 3.2 and from 12 to 1 after $24 \mathrm{~h}$ of cold treatment, respectively.

One 3-ketoacyl-CoA thiolase (KCT) gene, three 4-coumarate-CoA ligase(4CL) genes and one allene oxide cyclase $(A O C)$ genes in jasmonic acid (JA) biosynthesis pathways were identified in our dataset. KCT and 4CL genes were highly expressed with RPKM values $>100$ and were significantly down-regulated after $24 \mathrm{~h}$ of cold treatment. AOC gene, known as a key enzyme in JA biosynthesis, was significantly up-regulated by 10.3 and 9.2 folds at $24 \mathrm{~h}$ and $48 \mathrm{~h}$ of cold treatment, with an increased RPKM value from 0.024 to 0.251 and 0.223 , respectively..

\section{Validation of the differentially expressed genes (DEGs) via RT- qPCR.}

To verify the reliability and accuracy of this Illumina RNA-seq data, quantitative real-time reverse transcription-PCR (qRT-PCR) was used to assess the relative expression levels for 14 selected key genes of six endogenous hormone synthesis pathways in every treatment group. GAPDH was selected for internal control reference gene. The correlation between qRT-PCR and RNA-seq was measured by scatter plotting log2-fold changes, which showed a positive correlation coefficient in both techniques (Fig. 5). This independent evaluation revealed the reliability of the RNA-seq data.

\section{Influence of cold stress on endogenous hormone contents and hormone ratios}

The effect of cold stress on the endogenous hormone contents were examined. The dynamic changes in $A_{B A}, A_{1}$ +3 , IAA and ZR in three stages under $25^{\circ} \mathrm{C}$ or $0^{\circ} \mathrm{C}$ were shown in Fig. 6 . The result indicated that there were no significant differences in all the four endogenous hormone contents between $0-48 \mathrm{~h}$ under $25^{\circ} \mathrm{C}$. During $0^{\circ} \mathrm{Ccold}$ stress, the ABA content strongly increased by 1.75 - and 1.83-fold $(P<0.01)$ at 12 and $24 \mathrm{~h}$, compared with the control, respectively. And then gradually decreased to $25 \%$ higher than the control. $\mathrm{GA}_{1+3}$ levels increased to peak at $24 \mathrm{~h}$ by $161 \%$ and then maintained the higher level. The ratio of ABA to GA first skyrocketed to peak at $12 \mathrm{~h}$ by 
88.3\% and then decreased to a very low level. IAA content basically maintained constant. The ratio of ABA to IAA increased by $151.8 \%, 238.5 \%$ and $63.7 \%$ at 12 h, $24 \mathrm{~h}$ and $48 \mathrm{~h}$, respectively. On the contrary, ZR levels decreased significantly at $24 \mathrm{~h}$ by $42.8 \%(\mathrm{P}<0.05)$ and then maintained the low level. Accordingly, the elevated ratio of $A B A$ to ZR ranged from $121.5-395.8 \%$ under cold stress. The most significant increase was observed at $24 \mathrm{~h}$ by $395.8 \%$ as compared to the control.

In general, $A B A$ content was strongly induced by cold stress in the early stages, $\mathrm{GA}_{1+3}$ content was significantly higher in the middle-late stages, while ZR content was significantly lower, especially in the middle-late stages. The changes of $A B A / G A, A B A / I A A$, and $A B A / Z R$ ratio under cold stress showed an increasing trend in the early-middle stages, but then gradually decreased as the stress time prolonged.

\section{Effect of exogenous phytohormones and biosynthesis inhibitors on cold stress response}

A systematic pilot experiments were conducted by pot experiments to determine the effects of multiple plant growth regulators on cold stress response of T. hemsleyanum. The results showed that GA3 and ABA were the most important phytohormones affecting the stress related indicators.

After exogenous hormonal applications, the stress related indicators, including MDA content, soluble protein content and electrolyte leakage were simultaneously analyzed. As shown in Fig. 7A, ABA-treated seedlings had significantly more soluble protein content than control. ABA spraying dramatically decreased the MDA content and electrolyte leakage. The MDA content plummeted from $7.1 \pm 1.4 \mu \mathrm{mol} / \mathrm{g}(\mathrm{CK})$ to $5.1 \pm 1.1 \mu \mathrm{mol} / \mathrm{g}(\mathrm{ABA})$. The electrolyte leakage plummeted from $73.5 \pm 8.4 \%$ (CK) to $50.0 \pm 11.2 \%$ (ABA). FLU-treatment drastically increased electrolyte leakage and MDA content to $94.1 \pm 13.2 \%$ and $11.2 \pm 1.3 \mu \mathrm{mol} / \mathrm{g}$, respectively. By contrast, the combined use of ABA and FLU had relatively stable MDA content, electrolyte leakage, and soluble protein content. On the other hand, $\mathrm{GA}_{3}$-treatment had significant less soluble protein content than control. $\mathrm{GA}_{3}$ spraying dramatically increased the MDA content and electrolyte leakage. The MDA content soared from $7.1 \pm 1.4 \mu \mathrm{mol} / \mathrm{g}$ (CK) to $11.8 \pm 1.3 \mu \mathrm{mol} / \mathrm{g}$ (GA3). The electrolyte leakage soared from $73.5 \pm 8.4 \%$ (CK) to $92.3 \pm 16.2 \%$ (GA3). Compared with $\mathrm{GA}_{3}$-treated seedlings, the electrolyte leakage and MDA content were greatly decreased to $59.9 \pm$ $8.1 \%$ and $6.6 \pm 1.4 \mu \mathrm{mol} / \mathrm{g}$ in the presence of PAC, by $35.5 \%$ and $44.1 \%$, respectively. The combined use of $\mathrm{GA}_{3}$ and PAC had relatively stable MDA content and electrolyte leakage.

We found that treatment with ABA or GA can suppress or enhance chilling damage, respectively. However, we thought therefore that ABA-mediated suppression and GA-mediated enhancement of chilling damage could be reversed by the application of exogenous FLU and PAC, respectively.

The combined effects of GA and ABA on cold stress response were further tested (Fig. 7B C). GA-mediated promotion and $A B A$-mediated repression of chilling damage could both be attenuated by the co-application of $A B A$ and $\mathrm{GA}_{3}$, respectively, suggesting that the effects of $\mathrm{GA}_{3}$ and $A B A$ are antagonistic. Interestingly, the promotion of chilling damage by $\mathrm{GA}_{3}$ was only significantly attenuated when ABA was applied within $6 \mathrm{~h}$ of the initial $\mathrm{GA}_{3}$ treatment. No significant attenuation was seen if ABA was added at 12 or $24 \mathrm{~h}$ (Fig. 7B). Moreover, ABA-mediated alleviation of chilling damage was aggravated by supplementation with $\mathrm{GA}_{3}$ at 2 or $6 \mathrm{~h}$ after the initial application of ABA. No significant attenuation was seen if $\mathrm{GA}_{3}$ was added at 12 or $24 \mathrm{~h}$ (Fig. 7C). 


\section{Discussion}

In our previous study [13], we found that $0-48 \mathrm{~h}$ is the critical period of the chilling damage in T. hemsleyanum. After that time, serious injury or even death are inevitable. In the present study, the transcriptomes of T. hemsleyanum during $0-48 \mathrm{~h}$ of cold-treatment duration were compared by using the Illumina sequencing platform. Then GO and KEGG enrichment analysis of the DEGs were used to indicate the biological processes involved in response to cold stress. The results indicated that some biological process categories related to endogenous hormone response skyrocketed in the early stress stage, such as 'hormone-mediated signaling' 'response to hormone' and 'response to endogenous stimulus' in $12 \mathrm{~h}$ and $24 \mathrm{~h}$. Therefore, we speculated that phytohormones regulatory network was a significant mechanism in response to cold stress of T. hemsleyanum.

\section{Metabolic changes of endogenous hormones in response to cold stress}

Phytohormones, such as ABA, IAA, GA, and so on are known to play critical roles in the complex signaling cascades and also in adaptive responses to various biotic and abiotic stresses. The cooperation and antagonism among the various hormones are recognized to be existent objectively in the growth and development of plants, such as dormancy release and germination. However, it remains poorly understood whether such an antagonistic relationship exists in the regulation of plant stress response.

ABA is perhaps the most well characterized phytohormone of various stress signals. ABA biosynthesis is one of the fastest responses of plants to abiotic stress, triggering downstream genes expression and causing stomatal closure, thereby enhancing cell water retention and the content of osmotic adjustment substances, such as proline, soluble sugar, and soluble protein. This could ultimately lead to restricting cellular growth. There was a significant positive correlation between the ABA accumulation and the drought and cold tolerance in rice [14]. Exogenous ABA could improve the cold tolerance of plants [15]. In higher plants, some major genes for the enzymes in ABA biosynthetic pathway have been identified. The first step in the ABA biosynthesis pathway is catalyzed by a zeaxanthinepoxidase (ZEP), whose molecular identity was first revealed in tobacco [16]. The 9-cis epoxycarotenoid dioxygenase (NCED) has been suggested to be a key rate-limiting enzyme during ABA biosynthesis [17]. It was originally identified from maize viviparous 14 mutants, which cleaves the ABA precursor C40-cis- poxycarotenoids, 9-cis-neoxanthin, and so on to produce xanthoxin, the direct precursor of ABA [18]. NCED is a rate-limiting enzyme in ABA synthesis and various studies reported transcriptional regulation of NCED following abiotic stress [19]. In T. hemsleyanum, three ZEP genes were up-regulated by approximately 2.5-9.8 folds after $12 \mathrm{~h}$ of cold treatment, and two NCED genes were up-regulated by approximately 2.0-2.5 folds after $24 \mathrm{~h}$ of cold treatment. Up-regulation of these genes implied that synthesis of ABA might be elevated under cold, which is consistent with results reported previously in Zea mays ssp. mexicana $L$ [20] and rice[21]. It is notable that most of these ABA biosynthesis-related genes were suddenly down-regulated after $24 \mathrm{~h}$ or $48 \mathrm{~h}$ of cold treatment. Accordingly, the ABA content was also first strongly increased, continuously came up to a "peak value" at $24 \mathrm{~h}$, and then gradually decreased to a comparatively low level. This result is consistent with studies of Poncirus trifoliata [22] and P. euphratica[23]. It is suggested that the increased levels of expression of ABA-responsive genes and an increased ABA content would be beneficial for plants under environmental stress[24]. Thus, a series of related genes involved in ABA biosynthesis and signaling pathway of T. hemsleyanum were expressed at low levels after $48 \mathrm{~h}$ of cold treatment, which might be one factor contributing to cold-sensitivity of T. hemsleyanum. 
GAs, as a group of tetracyclic diterpene carboxylic acids, regulate many critical biological events in plants, such as stem elongation, seed germination, and so on. Among the different GAs identified from plants, a few act as endogenous plant growth regulators including $\mathrm{GA}_{1}, \mathrm{GA}_{3}$, and so on. $\mathrm{GA}_{3}$ is considered to be the earliest known cold-related phytohormone. Plant growth could be restrained partly by the accumulation of DELLA proteins, a critical component in GA signaling $[25,26]$, suggesting the plants could balance their growth and the resistance to abiotic stress though down-regulating GA levels [27].

Bioactive GAs are synthesized from geranyl geranyl diphosphate (GGDP), a common C20 precursor for diterpenoids [28]. The final stage of bioactive GA synthesis is catalyzed by GA20ox and GA3ox, respectively. GA1 content in rice would be reduced by the suppression of GA3ox2, or the activation of GA2ox1 and GA2ox3 [29]. GA20ox is a rate-limiting enzyme in GA synthesis and regulation pathway. Over-expression of GA20ox often leads to plant growth acceleration and internode elongation by promoting GA synthesis [30]. Researches indicated that it would be beneficial for plants to survive in adversity at the cost of growth loss. The inhibition of plant endogenous hormones, particularly auxin and GA, would dramatically increase the surviving ability of plant under environmental stress. This could be a proactive self-protection mechanism of plant [31]. Niu et al[32] reported that low temperature delayed the growth and development of tobacco adventitious root by inhibiting GA signal transduction and dramatically reducing GA content. Similarly, the GA20ox genes in Zea maize were also downregulated by cold treatment [33]. In our study, two GA200x genes were down-regulated significantly under cold stress, which is in accord with this theory that growth retardation is a typical evolution characteristic of plant adaptation to environmental stress. But there were still some opposite reports, GA20ox genes of citrus were stimulated by chilling treatment [34]. The enzymes involved in GA synthesis pathway of Thlaspi arvensi were induced by low temperature [35].

GA2ox could transform bioactive $\mathrm{GA}_{1}$ and $\mathrm{GA}_{3}$ to inactive $\mathrm{GA}_{8}$ and $\mathrm{GA}_{34}$ by catalyzing hydroxylation reactions at C2. Over-expression of GA2ox would inhibit GA synthesis, and thus it would lead to plant growth retardation. The up-regulation of GA20x genes was found to be concerned with the high tolerance to drought [36]. Similarly, in our study, six putative members of the GA2ox gene family were identified in T. hemsleyanum. Among them, four genes exhibited a similar expression profile and all were significantly up-regulated by approximately $3-7$ folds following cold treatment, with a peak at $12 \mathrm{~h}$ after exposure. But then their expression level declined rapidly with the extension of stress time.

On the other hand, GA3ox was a positively regulatory factor of GA synthesis. An A20/AN1 zinc finger protein negatively regulated plant height in rice by reducing expression of GA3ox, enhancing expression of GA2ox 1 and GA2ox3, and decreasing the level of $\mathrm{GA}_{1}$ [37]. In our study, a significantly up-regulated GA3ox was annotated, which was up-regulated by approximately 6 folds following cold treatment, with a peak at $24 \mathrm{~h}$ after exposure. Accordingly, GA content increased to peak at $24 \mathrm{~h}$ by $161 \%$ and then maintained at the higher level. Previous studies on Zea mays ssp. maize L. transcript profile under cold, salt, and drought stresses indicated that the abundance of bioactive GAs was reduced, suggesting a negative effects of GA in stress tolerance[20]. This is in disagreement with what we observed in cold-stressed T. hemsleyanum. The up-regulation of GA3ox (c78816_g1, C8451_g1 and c57017_g2) and the down-regulation of GA20ox (c98675_g1, c54888_g1, c93517_g2, c85773_g1) were induced by cold, suggesting an inconsistent role of GA in this response. Thus, we speculated that there was a more complicated genes network of GA biosynthesis involving in cold-induced responses, besides these conventional rate-limiting enzymes. Future more comparative study will provide better understanding of the molecular basis of the GA associated response to cold in T. hemsleyanum. 
Indole-3-acetic acid (IAA), the major auxin which has been recognized for involving in plant cell division, differentiation and so on. Recent reports suggested that auxin is also involved in stress responses. Transcript profiling of rice revealed that the expression of many auxin-related genes were regulated in response to dehydration stress [38]. With the increase of cold stress intensity, the content of IAA decreased significantly in roots of Sea buckthorn, which was a cold-tolerance plant species [39]. So far, the main IAA biosynthesis and regulation pathways were still largely unknown. Mashiguchi et al suggested that IAA is produced from IPA by the YUC family of flavin-containing monooxygenase (FMO)[40]. The OsGH3-2 overexpression rice showed increased resistance to cold stress due to the combined effects of reduced free IAA content, decreased membrane penetrability, and so on[14]. Our study was similar to those results, two FOM unigenes were identified in T. hemsleyanum transcriptome, and both them were down-regulated after $48 \mathrm{~h}$ of cold stress. But as for the content of IAA, although a slight increase was noted at $12 \mathrm{~h}$, the difference was not statistically significant, and then it remained basically stable. This could be because the expression levels of the two FOM genes were comparatively low, with RPKM values $<60$. So the effects of them on IAA metabolism should be diminished.

Jasmonates(JAs) are the important signal molecules that regulate and enhance resistance in plants to abiotic stress, such as cold stress, and biotic stress. The application of exogenous methyl jasmonate (MeJA) in Camellia japonica[41] and banana fruit [42] significantly enhanced cold tolerance by increasing endogenous JA accumulation during cold storage. A recent study showed that the JA signaling pathway plays an important role in the early cold response by modulating the CBF signaling pathway. In plants, Allene oxide synthases (AOCs) are known as key enzymes in JA biosynthesis. JA is synthesized from a-linolenic acid. AOS and allene oxide cyclase activity subsequently results in cis-(+)-12-oxo-phytodienoic acid (OPDA). AOC is required for formation of cis-(+)enantio-mer (9S, 13S) of OPDA, which is thought to be the precursor of JA. OPDA, JA, and their derivatives can stimulate AOC expression. Suppressing AOC1 expression in Medicago truncatula roots could lower JA levels significantly [43]. Biotic or abiotic stresses that lead to endogenous increases in JAs are usually accompanied by upregulation of $A O C$ and $A O S$ transcription [44]. Our results also showed that the expression of AOC genes encoding the main JA biosynthetic enzymes were up-regulated significantly, indicating a high level of JA in T. hemsleyanum during cold stress stage.

Ethylene (ET) is a growth regulator involved in leaf abscission, fruit ripening, growth of adventitious roots, and so on. Moreover, it is synthesized in response to stresses such as wounding, chilling, and freezing [45]. ET biosynthesis is originated from the amino acid methionine generated by the Yang cycle, in which the precursor Sadenosylmethionine (AdoMet) is converted into 1-aminocyclopropane-1-carboxylic acid (ACC) by the enzyme 1aminocyclopropane - 1-carboxylase synthase (ACS)[46]. ACO (I-aminocyclopropane-1-carboxylate oxidase), termed as ET synthase, can directly catalyze the conversion of ACC to ET. Also, the ACO would directly control the content of ET because of its effect in the final step of the pathway. An increase in levels of free ACC content was observed to coincide with an increase in ET production of the drought-tolerant soybean cultivar[47]. In the present study, the expression levels of ACO genes were increased dramatically under cold stress, yet simultaneously, a significant decrease in the transcript levels of ACS genes occurred in T. hemsleyanum. It is interesting to note that the expression trend of ACO and ACS genes were divergent. It can be deduced from the above information that the ET biosynthesis was terribly sensitive to cold stress. Both the ACO and ACS were members of the gene family which is termed as a responsive gene family to cold stress, characteristic with increase and decrease at the early stage, respectively. We supposed that when a short interval of cold stress was applied, ET biosynthesis was promoted briefly because of the overproduction of ACO, but when the stress duration was increased, ET biosynthesis would be increasingly suppressed because of the inactivation of ACS. Previous studies had showed

Page $10 / 29$ 
partial similarity to our works. Sun et al [45] reported that ET positively regulated cold tolerance in grapevine, the cold tolerance was reduced by the application of the ET biosynthesis inhibitor aminoethoxyvinylglycine but increased by the treatment of the ET precursor ACC. But on the contrary, Shi et al [48] reported that ET negatively regulated plant responses to freezing stress in Arabidopsis thaliana. Freezing tolerance was decreased by the application of the ET precursor ACC but increased by the addition of the ET biosynthesis inhibitor, and the ETinsensitive mutants displayed enhanced freezing tolerance. In general, these results suggested that the role of ET in cold tolerance varied in different species.

\section{The proportion change and interaction of various endogenous hormones in response to cold stress}

It is generally perceived that $A B A / G A$ ratio could affect seed germination and sex differentiation, $A B A / I A A$ ratio could affect growing potential, and ABA/ZA could affect stomatal oscillation. However, it remains much less understood whether such an antagonistic relationship exists in the regulation of plant stress response. Some studies showed that under environment stress, a variety of phytohormones promoted and restricted mutually to reach a new balance by inducing stomatal closure, weakening transpiration, and slowing down growing rate, which is tightly related with the plant's ability to withstand stress and disturbance.

ABA and GA were thought to be hormone partners that functioned via a complicated and antagonistic network in which transcription factors DREB [49] and OsAP2-39 [50] were found to play a critical role in rice. It has been reported that restricting GA-promoted processes would in turn to promote ABA accumulation in Arabidopsis [25]. In contrast, it was found that the biosynthesis of both GA and ABA were inhibited by an A20/AN1-type zinc finger protein, which led to the decreased stress tolerance of rice [51]. Some evidence at the transcriptional level demonstrated that GA and ABA had different and contrasting effects in a variety of developmental processes of plant [52]. ABA levels and the ratio of $A B A / G A$ in leaves of cold-tolerant bamboo species were both higher than those of cold-sensitive bamboo species for all cold treatments, while $\mathrm{GA}_{1}$ and $\mathrm{GA}_{4}$ were conversely lower [11]. Similarly, Huang et al [8] verified that exogenous ABA treatment could promote the increase of endogenous $A B A$ content and the ratio of $A B A / G A$, in contrast with the decreased contents of $M D A$ and $\mathrm{GA}_{3}$, and thereby induced cold stress tolerance of sugarcane seedling. In accordance with the previous reports, our study indicated that ratio of $A B A / G A$ in $T$. hemsleyanum was increased steeply by 1.9 -fold at $12 \mathrm{~h}$, but then declined sharply to about the level of CK.

Previous reports suggested that a crosstalk of the signaling pathway also occurred between the ABA and auxin responses. For example, $A B L 1$, an $A B A$ signaling component of rice could modulate auxin responses by regulating the expression level of ABRE-containing genes related to auxin metabolism [53]. Auxin response factor $2\left(\mathrm{ARF}_{2}\right)$ was revealed to play a role in the ABA signaling pathway [54]. IAA content was significantly declined in rice treated with the ABA biosynthesis inhibitor, and expression of auxin synthesis and metabolism-related genes were changed in the ABA-inhibited rice. Endogenous IAA levels were decreased in the carotenoid-deficient rice mutants, demonstrating that ABA and IAA could mutually affect each other at the biosynthesis level, and the increased resistance to cold stress was probably related to the deficiency in IAA [6]. In the current study, we found that endogenous IAA levels decreased slightly in the cold-treated T. hemsleyanum under cold stress, but the change was not significant. Ratio of ABA/IAA was increased gradually from $0 \mathrm{~h}$ to $24 \mathrm{~h}$, then declined at $48 \mathrm{~h}$, but the value was still higher than control. The increase amplitude of $A B A / G A$ ratio was greater than that of $A B A / I A A$, and

Page $11 / 29$ 
as well the peak time for enhancement of $A B A / G A$ ratio was earlier than that of $A B A / I A A$, which demonstrated that $A B A / G A$ had a greater impact on the stress response than ABA/IAA.

Few studies have been reported the relationship between $A B A$ and other phytohormones. Ratio of $A B A / G A_{3}$ was one of the important factors that could effectively increase cold stress tolerance in sugarcane plants, along with the increased ratio of $A B A / I A A$ and the ratio of $A B A / Z R$, and exogenous $A B A$ application reduced GA3 and ZR content but increased endogenous $A B A$ content [8]. Guo et al [55] reported that a combination of the increased ZT/IAA ratio and $\mathrm{H}_{2} \mathrm{O}_{2}$ concentration was the basis for the enhanced shoot morphogenesis in cold-treated Saussurea involucrata explants. In our study, ABA/ZR ratio in T. hemsleyanum also showed an obviously increasing trend, which was correlated with the increase of ABA content and the decrease of ZR content under prolonged cold stress. On this basis, it could be inferred that other phytohormones also could influence stress tolerance through cross-talk mechanisms.

\section{The antagonistic relationship of $\mathrm{ABA}$ and $\mathrm{GA}_{3}$ in response to cold stress}

Hormones co-regulate plant growth and stress response in a critical way, and they act either synergistically or antagonistically[37], among which ABA and GA were showed to be the most effective hormones in cold stress response of $\mathrm{T}$. hemsleyanum. It was verified that GA regulated a variety of critical biological events in plants, including petal growth and seed germination [52]. As a versatile regulator, ABA has been shown to act antagonistically to the function of GA in many developmental processes, including petal elongation [52] and seed germination [56]. However, it remains poorly understood whether such an antagonistic relationship exists in the regulation of stress response.

The higher the electrolyte leakage rate is, the more serious the damage by cold stress is. Besides, MDA content can reflect the severity of membrane lipid peroxidation and the stress tolerance of plants. So they are negatively correlated with stress tolerance ability of plants. The soluble proteins could play important roles in osmotic adjustment, and it is positively correlated with stress tolerance of plants. In this study, the electrolyte leakage rate and MDA content of the GA-treated plantlets were promoted, while the soluble protein content was reduced than control. And instead, ABA-treatment had contrary influences on these stress related indicators. Moreover, we also found that when the biosynthesis of endogenous ABA and GA were inhibited by FLU and PAC, respectively, the effects of GA and ABA treatment were reversed. The combined effects of GA and ABA on cold response were further tested. Two findings in our current study showed that GA and ABA had antagonistic effects on cold tolerance of T. hemsleyanum: (1) cold-endurance ability was enhanced by ABA but repressed by GA when each phytohormone was applied alone; (2) the ABA-mediated promotion and GA-mediated repression of cold tolerance could both be attenuated by the co-application of the two phytohormones, respectively. However, the antagonistic effect of GA and ABA was no longer apparent when the treatment was extended to longer than $6 \mathrm{~h}$, indicating that transcriptional regulation of hormone-associated pathways was an early and transient event during stress response.

\section{Conclusions}


In summary, the transcriptome of T. hemsleyanum during 0-48 h of cold-treatment indicated that phytohormones regulatory network was a significant mechanism in response to cold stress. Some hormone-related genes, such as NCED, ZEP, GA3ox, FOM, AOC and so on, were crucial enzyme genes, which resulted in the significant changes of hormones content. The response of $A B A$ and $A B A / G A_{1+3}$ to cold stress was prior to that of $G A_{1+3}, Z R, A B A / I A A$, and $A B A / Z R$. The increasing changes in $A B A / G A_{1+3}$ turned to a steep decline with the extension of stress time, which might be one factor contributing to cold-sensitivity of T. hemsleyanum under prolonged stress. Our data supported the hypothesis that GA and ABA could work antagonistically to balance the early responses to cold in T. hemsleyanum. This study also suggested that PAC, a GA biogenesis inhibitor, as well as exogenous ABA, might be used as early interventions for chilling damage of $T$. hemsleyanum. Further study in the relationship among ABA/GA homeostasis and the regulation of the stress-related genes involved in the phytohormone signaling network can provide a new clue for understanding their connection in stress response.

\section{Methods}

\section{Plant materials and cold stress treatments}

Healthy sterile test-tube plantlets of T. hemsleyanum were obtained and cultured as the same as our previous report [57]. Based on our preliminary work results of physiological parameters determination, the temperature and sampling time of cold stress treatment were optimized [13]. For both the control and cold stress tests, these seedlings were cultured in the same conditions except the different temperatures at $25^{\circ} \mathrm{C}$ or $0{ }^{\circ} \mathrm{C}$. After $0 \mathrm{~h}, 12 \mathrm{~h}$, $24 \mathrm{~h}$, and $48 \mathrm{~h}$ of cold stress treatment, the young leaves of 10 individual 4 week-old seedlings were collected and immersed in liquid nitrogen immediately and stored at $-80^{\circ} \mathrm{C}$ for RNA extraction and quantitative real-time PCR analysis. Three biological replicates were performed at the same time. Each biological replicate consisted of a pool of leaves from 10 seedlings.

\section{Rna Extraction, Quality Determination, And De Novo Assembly}

Total RNA was extracted with Trizol reagent (Invitrogen, CA, USA), following the manufacturer's protocol. RNA purity was checked using the kaiaoK5500®Spectrophotometer (Kaiao, Beijing, China). RNA integrity and concentration was assessed using the RNA Nano 6000 Assay Kit of the Bioanalyzer 2100 system (Agilent Technologies, CA, USA) at Annoroad Gene Technology (Beijing) co., LTD. The clustering was performed on a cBot cluster generation system using HiSeq PE Cluster Kit v4-cBot-HS (Illumina), and sequenced by Illumina HiSeq2500. The software Trinity was used for de novo assembly which was developed at the Broad Institute and the Hebrew University of Jerusalem. Trinity partitions the sequence data into many individual de Bruijn graphs, each representing the transcriptional complexity at a given gene or locus. The clean data were mapped to the assembled transcripts by Bowtie 2(v2.2.3).

\section{Coding Regions Prediction And Transcriptome Functional Annotation}

The candidate coding regions within transcript sequences, such as those generated by de novo RNA-Seq transcript assembled by Trinity were identified by TransDecoder. The functional annotation of unigenes and ORFs were performed by Trinotate. 


\section{Rpkm Estimation And Deg Analysis}

Reads Count for each gene in each sample was counted by HT Kilobase Millon Mapped Reads each sample, the formula is shown as:

\section{$\mathrm{RPKM}=10^{6 * \mathrm{R} /\left(\mathrm{NL} / 10^{3}\right)}$}

$\mathrm{R}$ is the number of reads in a certain sample that is assigned to a certain gene, $\mathrm{N}$ is the total number of mapped reads in the certain sample and $L$ is the length of the certain gene.

The differential gene expression between samples was analyzed by DESeq(v1.16). P-value could be assigned to each gene and adjusted by the Benjamini and Hochberg's approach for controlling the false discovery rate. " $q \leq$ 0.05 and |log2_ratiol $\geq 1$ " was set as the threshold to determine the differentially expressed genes (DEGs).

\section{Functional Annotation And Classification Of Degs}

The GO (Gene Ontology, http://geneontology.org/) enrichment of DEGs was implemented by hypergeometric test. $\mathrm{GO}$ terms with $\mathrm{q}<0.05$ were considered to be significantly enriched. KEGG (Kyoto Encyclopedia of Genes and Genomes http://www.kegg.jp/) analysis of the DEGs was performed to identify the associated biochemical and signal transduction pathways. KEGG terms with $\mathrm{q}<0.05$ were considered to be significantly enriched.

\section{Validation Of Rna-seq Data By Qrt-pcr}

Quantitative real-time RT-PCR (qPCR) was performed to examine expression patterns of the candidate unigenes using a SteponePlus real-time PCR system (Applied Biosystems, Forster City, CA, USA). The primers for amplification are listed in Table 1. Each reaction contained $2 \mu \mathrm{l}$ of cDNA, $10 \mu \mathrm{l}$ of $2 x$ Power SYBR green PCR master mix (Applied Biosystems, Forster City, CA, USA), and $2 \mu$ of forward and reverse primers in a final volume of $20 \mu \mathrm{l}$. The PCR reactions were conducted by incubation at $95^{\circ} \mathrm{C}$ for 3 min followed by 40 cycles of $95^{\circ} \mathrm{C}$ for $15 \mathrm{sec}$ and $60^{\circ} \mathrm{C}$ for $45 \mathrm{sec}$. The GAPDH gene was used as the reference gene to normalize target gene expression. The calculation for relative gene expression was determined by the $2^{-\Delta \Delta C t}$. Dissociation-curve analysis was carried out to confirm the amplification specificity. Three technical replicates were performed for each sample and the data are shown as means \pm standard errors $(S E)(n=3)$.

\section{Determination Of Phytohormones Content By Elisa}

The double-antibody sandwich ELISA was performed as our previously described [54] with slight modifications for the endogenous phytohormones quantification. Briefly, $50 \mathrm{mg}$ of fresh leaves from $\mathrm{T}$. hemsleyanum seedlings were ground and then subjected to extraction ( $80 \%$ methanol containing $10 \mathrm{mg} / \mathrm{L}$ butylhydroxytoluene $\mathrm{v} / \mathrm{v}$ ) for $18 \mathrm{~h}$ at $4{ }^{\circ} \mathrm{C}$, and then centrifuged at $4000 \mathrm{rpm}$ for $15 \mathrm{~min}$ at $4{ }^{\circ} \mathrm{C}$. The supernatant was passed through $\mathrm{C} 18$ solid phase extraction cartridge (Waters Corporation, Millford, MA, USA) to remove the chlorophyll. The products were dried by pure $\mathrm{N}_{2}$ at $20^{\circ} \mathrm{C}$, and resuspended in $20 \mu \mathrm{l}$ cold solution from the kit for phytohormones-testing (MLBIO, Shanghai El Biotechnology Co., Ltd. China). Endogenous phytohormones levels were determined according to the manufacturer's instructions, respectively. The imark microplate reader (Bio-Rad Co. Ltd., Hercules, USA) was used to measure the ABA, IAA, ZR and GA content at $450 \mathrm{~nm}$ of the optical density (OD). 


\section{Hormone And Inhibitor Treatments}

For the evaluation of hormonal mediation in cold response, GA and/or ABA treatments were employed in the in vivo experiments. The young leaves of 10 individual seedlings were included for each treatment. In vivo treatments were performed by spraying with $5 \mathrm{ml} 20 \mu \mathrm{M}$ GA3 or $100 \mu \mathrm{M}$ ABA at $6 \mathrm{~h}$, then were sampled after $48 \mathrm{~h}$. As a control, seedlings sprayed with $0.1 \%$ ethanol in deionized water were sampled in parallel. The widely used inhibitors of GA and ABA biosynthesis, paclobutrazol (PAC) and fluridone (FLU), were also sprayed in this study. In the experiment protocol described above, $10 \mu \mathrm{M}$ PAC or $0.1 \mu \mathrm{M}$ FLU were sprayed with or without the phytohormones.

To evaluate the dynamic interaction between the effects of GA and $A B A$, the experiment using a combination of GA and ABA was further performed, in which, after spraying with ABA for 2, 6, 12, or $24 \mathrm{~h}$, GA was sprayed, with the final measurements being performed at $48 \mathrm{~h}$. Conversely, where GA was the initial hormone sprayed, ABA was then added during the experiment. Three biological replicates were performed at the same time. Each biological replicate consisted of a pool of leaves from 10 seedlings.

\section{Physiological Parameters Determination}

MDA content was measured as our previous report[13]. MDA content $(\mu \mathrm{mol} / \mathrm{g})=\left(\mathrm{C}_{\mathrm{MDA}} \times \mathrm{Vr} \times 10^{-3} \times \mathrm{Vt}\right) \times(\mathrm{W} \times \mathrm{Vs})^{-1}$. $\left.C_{M D A}=6.45\left(A_{532}-A_{600}\right)-0.56 A_{450}, \mu \mathrm{mol} / L\right) ; V r$ reaction volume $(\mathrm{mL})$; Vt: total volume of total extractive enzyme $(\mathrm{mL})$, Vs: tested enzyme volume $(\mathrm{mL}) ; \mathrm{W}$ : the weight of leaves $(\mathrm{g})$.

Electrolyte leakage was determined as our previous report [13]. The fresh leaves $(1 \mathrm{~g})$ were incubated with $15 \mathrm{ml}$ deionized water at $25^{\circ} \mathrm{C}$ for $24 \mathrm{~h}$. The electrolyte leakage was expressed as percent $(E L \%)=E_{1} / E_{2} \times 100 \%$. Deionized water without leaves incubated in it was taken as control.

Soluble protein content was measured by Coomassie Brilliant Blue G-250 staining. Soluble protein content (mg/g) $=\mathrm{C} \times \mathrm{Vt} \times(\mathrm{Vs} \times \mathrm{W} \times 1000)^{-1} ; \mathrm{C}$ : protein quantity determined from the standard curve $(\mu \mathrm{g})$; Vs: sample solution volume $(\mathrm{mL})$; Vt: total volume of the extracted solution $(\mathrm{mL})$; W: the weight of samples $(\mathrm{g})$.

\section{Statistical Analysis}

All data were collected from three biological replicates and three technical replicates. Values are presented as mean \pm SD of three independent experiments. All statistical analyses were performed using the SPSS 21.0 software (SPSS, Chicago, IL, USA). The difference among the multiple groups was analyzed by one-way analysis of variance followed by LSD multiple comparison tests. $\mathrm{P}<0.05$ was considered significant.

\section{Abbreviations}


Table 1

List of primer sequences of randomly selected unigenes used for qRT-PCR analysis

\begin{tabular}{|c|c|c|c|c|}
\hline GenelID & Gene Name & $\mathrm{Tm}$ & PrimernF区(5'-3') & PrimerDR】(5'-3')凹 \\
\hline c81504_g1 & ZEP_4 & 55.8 & TACAGTTTATTTAGCCCGCC & TTTCCTACTCGTTTCCATCC \\
\hline c102056_g1 & AA03_2 & 53.7 & AACGGTGAGTTGTGGTGGTG & GAAGGGTGGATGGTGGAGAC \\
\hline c66096_g3 & NCED_3 & 56.9 & САССТTСТGСТTССАССТС & TTTCTCCTCGCATTCGTTA \\
\hline c94964_g1 & AA01_3 & 54.8 & TAGAGTCCAACAGCAGAGCAG & GGAAGGGAGATAGTAAAGAAGTG \\
\hline c72906_g1 & CRMP_LOG1 & 55.0 & AGACAGTTGGGGAAGTGAAGG & TTATCTATGAATGAGAGGAGGGAG \\
\hline c73984_g2 & CRMP_LOG7 & 51.2 & АААТАССТСССТТСССТССТ & GCCTACACTTCCTCCACCAT \\
\hline c80750_g2 & CRMP_LOG8 & 56.5 & CTTCTTGCTTGGGTTATTTGAC & AGTTTATGGCGTCGGGTTC \\
\hline c100822_g2 & ACO_1 & 55.3 & CTCACCAAACAATGCTССТTC & TTTTCTTGATTTCCAACCCAG \\
\hline c90679_g2 & ACO_4 & 52.5 & ACTGTTTGTGTCACCACTCTATG & GTCAGCAACTACCCTCCСT \\
\hline c99260_g1 & ACS_1 & 56.0 & TTTTTTGACGGGTATGGTAGAG & GATTTGAAGGATTGGTGATGAG \\
\hline c93517_g2 & GA20ox_2 & 52.0 & AATGGGGTTGTTTCAGAGTC & AAAGAGGATTGATGTCGCTG \\
\hline c92581_g1 & GA20x_4 & 56.4 & TGGGGGGAGTTTGAGAGG & GCGTGAAGCATAGGGTGTTG \\
\hline c90130_g1 & KCT & 55.8 & CTTCTTCTTCGCCTTCTCGT & ACAACCACAATGTCATCCCC \\
\hline c52785_g1 & $\mathrm{AOC}$ & 56.0 & CAGATCCCCGCCGTGAT & GCGCCAAGCAGAGCCAG \\
\hline
\end{tabular}




\begin{tabular}{|c|c|c|c|}
\hline Abbreviation & Full name & Abbreviation & Full name \\
\hline ABA & abscisic acid & NCED & 9-cis-epoxycarotenoid dioxygenase \\
\hline GA & gibberellic acid & TatC & sec-independent protein translocase protein \\
\hline CK & cytokinin & IPT & adenylate isopentenyltransferase \\
\hline ZR & zeatin riboside & CRMP & $\begin{array}{l}\text { cytokinin riboside } 5^{\prime} \text {-monophosphate } \\
\text { phosphoribohydrolase }\end{array}$ \\
\hline PAC & paclobutrazol & STM & homeobox protein Shoot Meristemless \\
\hline ET & ethylene & YUCCA & indole-3-pyruvate monooxygenase \\
\hline JA & jasmonic acid & ACC & aminocyclopropane carboxylate oxidase \\
\hline BR & brassinosteroid & ACS & 1-aminocyclopropane-1-carboxylate synthase \\
\hline FLU & fluridone & DRBP & dehydration-responsive element-binding protein \\
\hline AAO3 & $\begin{array}{l}\text { abscisic-aldehyde } \\
\text { oxidase }\end{array}$ & CTR1 & serine/threonine-protein kinase \\
\hline CESA & cellulose synthase A & AA01 & indole-3-acetaldehyde oxidase \\
\hline ZEP & zeaxanthin epoxidase & ADLT & AMP dimethylallyltransferase \\
\hline EUPL & $\begin{array}{l}\text { E3 ubiquitin-protein } \\
\text { ligase }\end{array}$ & $\mathrm{CODH}$ & copal-8-ol diphosphate hydratase \\
\hline ELP & ET01-like protein & ECDS & ent-copalyl diphosphate synthase \\
\hline LOX2S & lipoxygenase & EKAH & ent-kaurenoic acid hydroxylase \\
\hline AOC & allene oxide cyclase & GA20ox & gibberellin 20-oxidase \\
\hline $4 \mathrm{CL}$ & $\begin{array}{l}\text { 4-coumarate-CoA } \\
\text { ligase }\end{array}$ & GA2ox & gibberellin 2-beta-dioxygenase \\
\hline EKES & $\begin{array}{l}\text { Ent-kaur-16-ene } \\
\text { synthase }\end{array}$ & GA3ox & gibberellin 3-beta-dioxygenase \\
\hline ATH1 & $\begin{array}{l}\text { homeobox protein } \\
\text { ATH1 }\end{array}$ & $\mathrm{ACO}$ & 1-aminocyclopropane-1-carboxylate oxidase \\
\hline ACDD & $\begin{array}{l}\text { acyl-CoA } \\
\text { dehydrogenase }\end{array}$ & CRMP_LOG & $\begin{array}{l}\text { Cytokinin riboside } 5 \text {-monophosphate } \\
\text { phosphoribohydrolase LOG }\end{array}$ \\
\hline KCT & $\begin{array}{l}\text { 3-ketoacyl-CoA } \\
\text { thiolase }\end{array}$ & & \\
\hline
\end{tabular}

\section{Declarations}

Ethics approval and consent to participate: Not applicable

Consent to publish: All Authors have read the manuscript and have agreed to submit it in its current form for consideration for publication. 
Availability of data and materials: The datasets generated during the current study are available in Supplementary files 1-7 were unigenes expression matrix data of ABA, Auxin, GA, Ethylene, JA, Cytokinin, and Brassinosteroid synthesis pathways in T. hemsleyanum transcriptome, respectively.

Competing interests: The authors declare that they have no conflict of interest.

Funding: The Natural Science Foundation Committee of Zhejiang province designed and published the project requirements guideline relevant to this research. This work was supported by National Natural Science Foundation of China Grants(81903743), Zhejiang province public welfare technology application research project (2017C32075), China postdoctoral science special foundation (2019T120552), China postdoctoral science foundation (2018M642554), and Ningbo city science and technology innovation 2025 major research project(2019B10008).

Author Contributions: Peng X conceived the study. Li M J performed GO and KEGG Pathways enrichment analysis; Wu H performed physiological indicators determination; Qiu W Y performed endogenous hormone determination; Chen $\mathrm{H} J$ performed RNA Extraction and quality determination. Peng $X$ wrote the manuscript, designed the experiments; Zhang Z Y carried out the analysis.

Acknowledgements: Not applicable.

\section{References}

1. Ru, Y., Chen, X., Wang, J., et al. Polysaccharides from Tetrastigma hemsleyanum Diels et Gilg: Extraction optimization, structural characterizations, antioxidant and antihyperlipidemic activities in hyperlipidemic mice. Int J Biol Macromol. 2019; 125: 1033-104

2. Peng, X., Zhang, Y.Y., Wang, J., et al. Ethylacetate Extract from Tetrastigma hemsleyanum induces apoptosis via the mitochondrial-caspase-dependent intrinsic pathway in $\mathrm{HepG}_{2}$ cells. Tumor Biol. 2016; 37: 865-876

3. Fu, J., Miao, Y., Shao, L., et al. De novo transcriptome sequencing and gene expression profiling of Elymus nutans under cold stress. BMC Genomics. 2016; 17: 870-889.

4. Xu, W., Li, R., Zhang, N. et al. Transcriptome profiling of Vitis amurensis, an extremely cold-tolerant Chinese wild Vitisspecies, reveals candidate genes and events that potentially connected to cold stress. Plant Mol Biol. 2014; 86: 527-541.

5. Yang, F.X., Zhu, G.F., Wei, Y., et al. Low-temperature-induced changes in the transcriptome reveal a major role of CgSVP genes in regulating flowering of Cymbidium goeringii. BMC Genomics. 2019; 20(1):53-68

6. Du, H., Wu, N., Chang, Y. et al. Carotenoid deficiency impairs ABA and IAA biosynthesis and differentially affects drought and cold tolerance in rice. Plant Mol Biol. 2013; 83: 475-488.

7. Sharma, S.,Chen,C., Khatri,K., et al. Gracilaria dura extract confers drought tolerance in wheat by modulating abscisic acid homeostasis. Plant physiol bioch. 2019; 136: 143-154.

8. Huang, X., Chen, M. H., Yang, L. T..Effects of Exogenous Abscisic Acid on Cell Membrane and Endogenous Hormone Contents in Leaves of Sugarcane Seedlings under Cold Stress. Sugar Tech. 2015; 17(1):59-64

9. Liu, X., Zhang, H., Zhao, Y., et al. Auxin controls seed dormancy through stimulation of abscisic acid signaling by inducing ARF-mediated ABI3 activation in Arabidopsis. P Natl Acad Sci. 2013; 10 (38): 15485-90 
10. Wang, Y., Li, B., Du, M., et al. Mechanism of phytohormone involvement in feedback regulation of cotton leaf senescence induced by potassium deficiency. J Exp Bot. 2012; 63(16): 5887-5901

11. Zhang, F., Wan, X. Q., Zhang, H. Q., et al. The effect of cold stress on endogenous hormones and CBF1 homolog in four contrasting bamboo species. J For Res. 2012; 17: 72-78

12. Yang, Q., Zhang, Z., Rao, J., et al.Low-temperature conditioning induces chilling tolerance in'Hayward' kiwifruit by enhancing antioxidant enzyme activity and regulating endogenous hormones levels. J Sci Food Agr. 2013; 93(15): 3691-3699

13. Peng, X., Wu, H., Chen, H.J., et al. Transcriptome profiling reveals candidate flavonol-related genes of Tetrastigma hemsleyanum under cold stress. BMC Genomics. 2019; 20: 687-702.

14. Du, H., Wu, N., Fu, J., et al. A GH3 family member, OsGH3-2, modulates auxin and abscisic acid levels and differentially affects drought and cold tolerance in rice. J Exp Bot. 2012; 63: 6467-6480

15. Cheng, Z.M., Jin, R., Cao, M.J., et al. Exogenous application of ABA mimic 1 (AM1) improves cold stress tolerance in bermudagrass (Cynodon dactylon). Plant Cell Tiss Organ Cult. 2016; 125: 231-240.

16. Park, H.Y., Seok, H.Y., Park, B.K., et al. Overexpression of Arabidopsis ZEP enhances tolerance to osmotic stress. Biochem Bioph Res Co. 2008; 375: 80-85.

17. Gonzalez, S.N., Lopez, L.I.M., Reyes, L.R., et al. The dioxygenaseen coding OLSD gene from Burkholderia cenocepacia causes the hydroxylation of the amide-linked fatty acyl moiety of ornithine-containing membrane lipids. Biochemistry. 2011; 50: 6396-6408

18. Boursiac, Y., Léran, S., Corratgé-Faillie, C., et al. Review: ABA transportand transporters. Trends Plant Sci. 2013; 18:325-333

19. Espasandin, F.D., Maiale, S.J., Calzadilla, P., et al. Transcriptional regulation of 9-cis-epoxycaro tenoid dioxygenase (NCED) gene by putrescine accumulation positively modulates ABA synthesis and drought tolerance in Lotus tenuis plants. Plant Physiol Biochem. 2014; 76:29-35.

20. Lu, X., Zhou, X., Cao, Y., et al. RNA-seq Analysis of Cold and Drought Responsive Transcriptomes of Zea mays ssp. mexicana L. Front. Plant Sci. 2017; 8: 136-151

21. Du, H., Wu, N., Chang, Y., et al. Carotenoid deficiency impairs ABA and IAA biosynthesis and differentially affects drought and cold tolerance in rice. Plant Mol Biol. 2013; 83:475-488

22. Wang, M., Zhang, X.N., Liu, J.H Deep sequencing-based characterization of transcriptome of trifoliate orange (Poncirus trifoliata (L.) Raf.) in response to cold stress. BMC Genomics. 2015; 16: 555-574.

23. Chen, J., Tian, Q., Pang, T., et al. Deep-sequencing transcriptome analysis of low temperature perception in a desert tree, Populus euphratica. BMC Genomics. 2014; 15: 326

24. Luo, C., Guo, C., Wang, W., et al. Overexpression of a new stress-repressive gene OsDSR2 encoding a protein with a DUF966 domain increases salt and simulated drought stress sensitivities and reduces ABA sensitivity in rice. Plant Cell Rep. 2014; 33: 323-336

25. Achard, P., Gong, F., Cheminant, S., et al. The cold-inducible CBF1 factor-dependent signaling pathway modulates the accumulation of the growth-repressing DELLA proteins via its effect on gibberellin metabolism. Plant Cell. 2008; 20:2117-2129.

26. Zhou, M., Chen, H., Wei, D., et al. Arabidopsis CBF3 and DELLAs positively regulate each other in response to low temperature. Sci Rep.2017; 7: 39819

27. Zentella, R., Zhang, Z., Park, M., et al. Global analysis of della direct targets in early gibberellin signaling in Arabidopsis. Plant Cell. 2007; 19:3037-3057. 
28. Sakamoto, T., Miura, K., Itoh, H., et al. An overview of gibberellin metabolism enzyme genes and their related mutants in rice. Plant Physiology. 2004; 134: 1642-1653

29. Liu, Y., Xu, Y., Xiao, J., et al. OsDOG, a gibberellin-induced A20/AN1 zinc-finger protein, negatively regulates gibberellin-mediated cell elongation in rice. J Plant Physiol. 2011; 168:1098-1105

30. Colebrook, E.H., Thomas, S.G., Phillips. A.L., et al. The role of gibberellin signalling in plant responses to abiotic stress. J Exp Biol. 2014; 217: 67-75

31. Achardp, Cheng H, De grauwel,Decat, J, Schoutteten, $H$, et al. Integration of plant responses to environmentally activated phyto hormonal signals. Science. 2006; 311(5757): 91-94

32. Niu, S.H., Li, Z., Yuan, H., et al. Proper gibberellin localization in vascular tissue is required to regulate adventitious root development in tobacco. J Exp Bot. 2013; 64(11):3411-3424

33. Shan, X.H., Li, Y.D., Jiang, Y., et al. Transcriptome profile analysis of maize seedlings in response to highsalinity, drought and cold stresses by deep sequencing. Plant Mol. Biol. Rep. 2013; 31:1485-1491.

34. Ana, M.V., Waddi, B.C., Manuel, T., et al.. García-Martínez. Regulation of gibberellin 20-oxidase gene expression and gibberellin content in citrus by temperature and citrus exocortis viroid. Planta. 2003; 217:442-448.

35. Hazebroek, J.P., Metzger, JD., Mansager, E. R.. Thermo inductive regulation of gibberellin in metabolism in Thlaspi arvensi. L II: Cold induction of enzyme in gibberellin biosynthesis. Plant Physiol. 1993; 102:547-552.

36. Li, K.Q., Xu, X.Y., Huang, X.S.,. Identification of Differentially Expressed Genes Related to Dehydration Resistance in a Highly Drought-Tolerant Pear, Pyrus betulaefolia, as through RNA-Seq. PLOS one. 2016; 11(2):e0149352.

37. Zhang, S.W., Zhang, D., Fan, S., et al. Effect of exogenous GA3 and its inhibitor paclobutrazol on floral formation, endogenous hormones, and flowering-associated genes in'Fuji' apple (Malus domestica Borkh.). Plant Physiol Bioch. 2016; 107:178-186.

38. He,C., Gao,G., Zhang,J., et al.Proteome profiling reveals insights into cold- tolerant growth in sea buckthorn. Proteome Sci. 2016; 14: 14

39. Mashiguchi, K., Tanaka, K., Sakai, T.,et al. The main auxin biosynthesis pathway in Arabidopsis. Proc Natl Acad Sci USA. 2011; 108:18512-18517.

40. Jain, M., and Khurana, J. P. Transcript profiling reveals diverse roles of auxin-responsive genes during reproductive development and abiotic stress in rice. Febs J. 2009; 276:3148-3162.

41. Li, Q., Lei, S., Du, K., et al. RNA-seq based transcriptomic analysis uncovers a-linolenic acid and jasmonic acid biosynthesis pathways respond to cold acclimation in Camellia japonica. Sci Rep. 2016; 6: 36463

42. Zhao, M. L., Wang, J. N., Shan, W., et al. Induction of jasmonate signalling regulators mamyc2s and their physical interactions with maice 1 in methyl jasmonate-induced chilling tolerance in banana fruit. Plant Cell Environ. 2012; 36 (1): 30-51

43. Isayenkov, S., Mrosk, C., Stenzel, I., et al. Suppression of allene oxide cyclase in hairy roots of Medicago truncatula reduces jasmonate levels and the degree of mycorrhization with Glomus intraradices. Plant physiol. 2005; 139: 1401-1410.

44. Wang, L., Zhu, Y., Hu, W., et al. Comparative Transcriptomics Reveals Jasmonic Acid-Associated Metabolism Related to Cotton Fiber Initiation. PLOS one. 2015; 10(6): e0129854.

45. Sun, X.,Zhao ,T., Gan, S., et al. Ethylene positively regulates cold tolerance in grapevine by modulating the expression of Ethylene response factor 057.Sci Rep. 2016; 6: 24066. 
46. Roje, S. S-adenosyl-L-methionine: Beyond the universal methyl group donor. Phytochemistry. 2006; 67(15): 1686-1698

47. Arraes, F. B., Beneventi, M. A., Lisei de Sa, M. E., et al. Implications of ethylene biosynthesis and signaling in soybean drought stress tolerance. BMC Plant Biol. 2015; 15:213-132

48. Shi, Y., Tian, S., Hou, L., et al. Ethylene signaling negatively regulates freezing tolerance by repressing expressing of CBF and type-A ARR genes in Arabidopsis. Plant Cell. 2012; 24: 2578-2595.

49. Li, J., Sima, W., Ouyang, B., et al. Tomato SLDREB gene restricts leaf expansion and internode elongation by downregulating key genes for gibberellin biosynthesis. J Exp Bot. 2012; 63: 6407-6420.

50. Yaish, MW., El-Kereamy, A., Zhu, T., et al. The APETALA-2-like transcription factor OsAP2-39 controls key interactions between abscisic acid and gibberellin in rice. PLoS Genet. 2010; 6: e1001098

51. Zhang, Y., Lan, H.X., Shao, Q.L. An A20/AN1-type zinc finger protein modulates gibberellins and abscisic acid contents and increases sensitivity to abiotic stress in rice (Oryza sativa). J Exp Bot. 2016; 67: 315-326.

52. Li, L., Zhang, W., Zhang, L., et al. Transcriptomic insights into antagonistic effects of gibberellin and abscisic acid on petal growth in Gerbera hybrida. Front. Plant Sci. 2015; 6: 168-181.

53. Yang, X., Yang, Y.N., Xue, L.J., et al. Rice ABI5-Like1 regulates abscisic acid and auxin responses by affecting the expression of ABRE-containing genes. Plant Physiol. 2011; 156: 1397-1409

54. Wang, L., Hua, D., He, J., et al. Auxin Response Factor2 (ARF2) and its regulated homeodomain gene HB33 mediate abscisic acid response in Arabidopsis. PLoS Genet. 2011; 7: e1002172.

55. Guo, B., Stiles, A.R., Liu, C. Z. Changes in endogenous hormones and oxidative burstas the biochemical basis for enhanced shoot organogenesis in cold-treated Saussurea involucrata explants. Acta Physiol Plant. 2013; 35:283-287

56. Hu, Y., Gao, Y.R., Wei, W., et al. Strawberry MOTHER OF FT AND TFL 1 regulates seed germination and postgermination growth through integrating GA and ABA signaling in Arabidopsis. Plant Cell Tiss Organ Cult. 2016; 126: 343-352

57. Peng, X., Zhang, T. T., Zhang, J. Effect of subculture times on genetic fidelity, endogenous hormone level and pharmaceutical potential of Tetrastigma hemsleyanum callus. Plant Cell Tiss Org. 2015; 122 (1): 67-77

\section{Supplementary Files Legend}

Additional files

Supplementary files 1 was unigenes expression matrix data of ABA synthesis pathways in T. hemsleyanum transcriptome.

Supplementary files 2 was unigenes expression matrix data of Auxin synthesis pathways in T. hemsleyanum transcriptome.

Supplementary files 3 was unigenes expression matrix data of GA synthesis pathways in T. hemsleyanum transcriptome.

Supplementary files 4 was unigenes expression matrix data of Ethylene synthesis pathways in T. hemsleyanum transcriptome.

Supplementary files 5 was unigenes expression matrix data of JA synthesis pathways in T. hemsleyanum transcriptome.

Supplementary files 6 was unigenes expression matrix data of Cytokinin synthesis pathways in T. hemsleyanum transcriptome. 
Supplementary files7 was unigenes expression matrix data of Brassinosteroid synthesis pathways in T. hemsleyanum transcriptome.

\section{Figures}

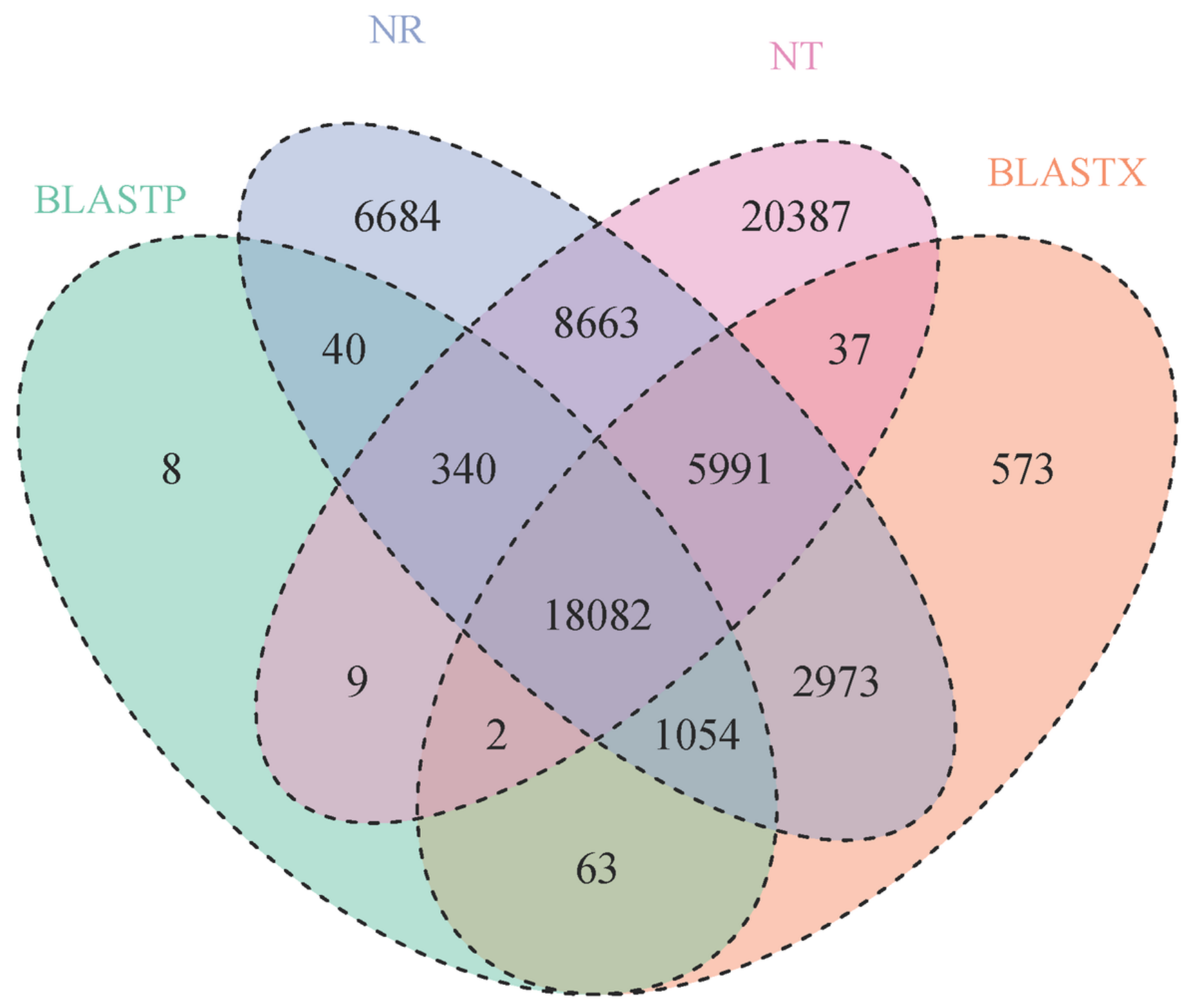

\section{Figure 1}

Venn diagram showing distribution of similar annotations from Nr, NT, BLASTX, and BLASTP databases. The overlapping regions among the four circles contain the number of unigenes that share similar hits with respective databases. 


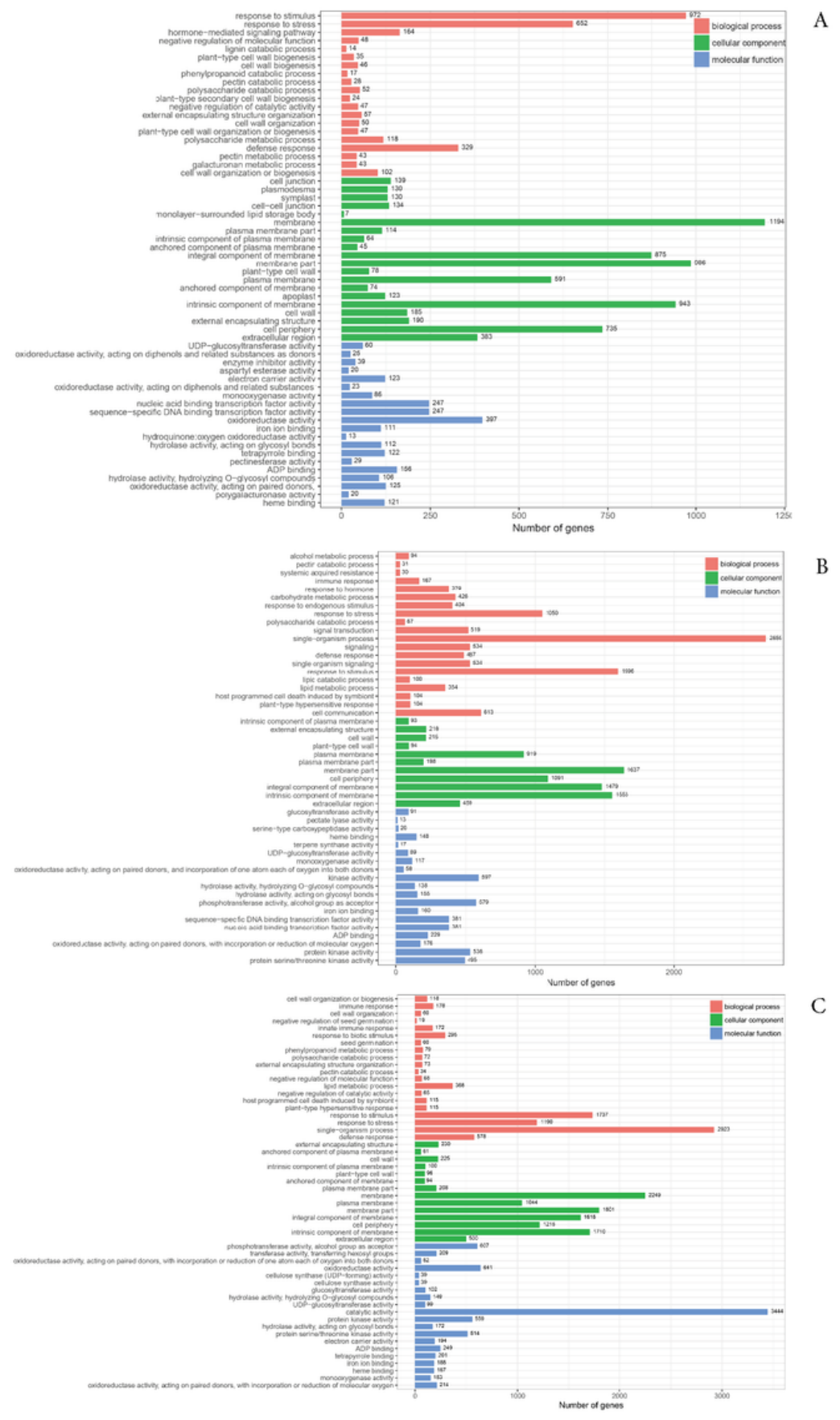

Figure 2

GO classifications of differentially expressed unigenes of T. hemsleyanum in response to $12 \mathrm{~h}(\mathrm{~A}), 24 \mathrm{~h}(\mathrm{~B})$, and $48 \mathrm{~h}(\mathrm{C})$ of cold exposure 


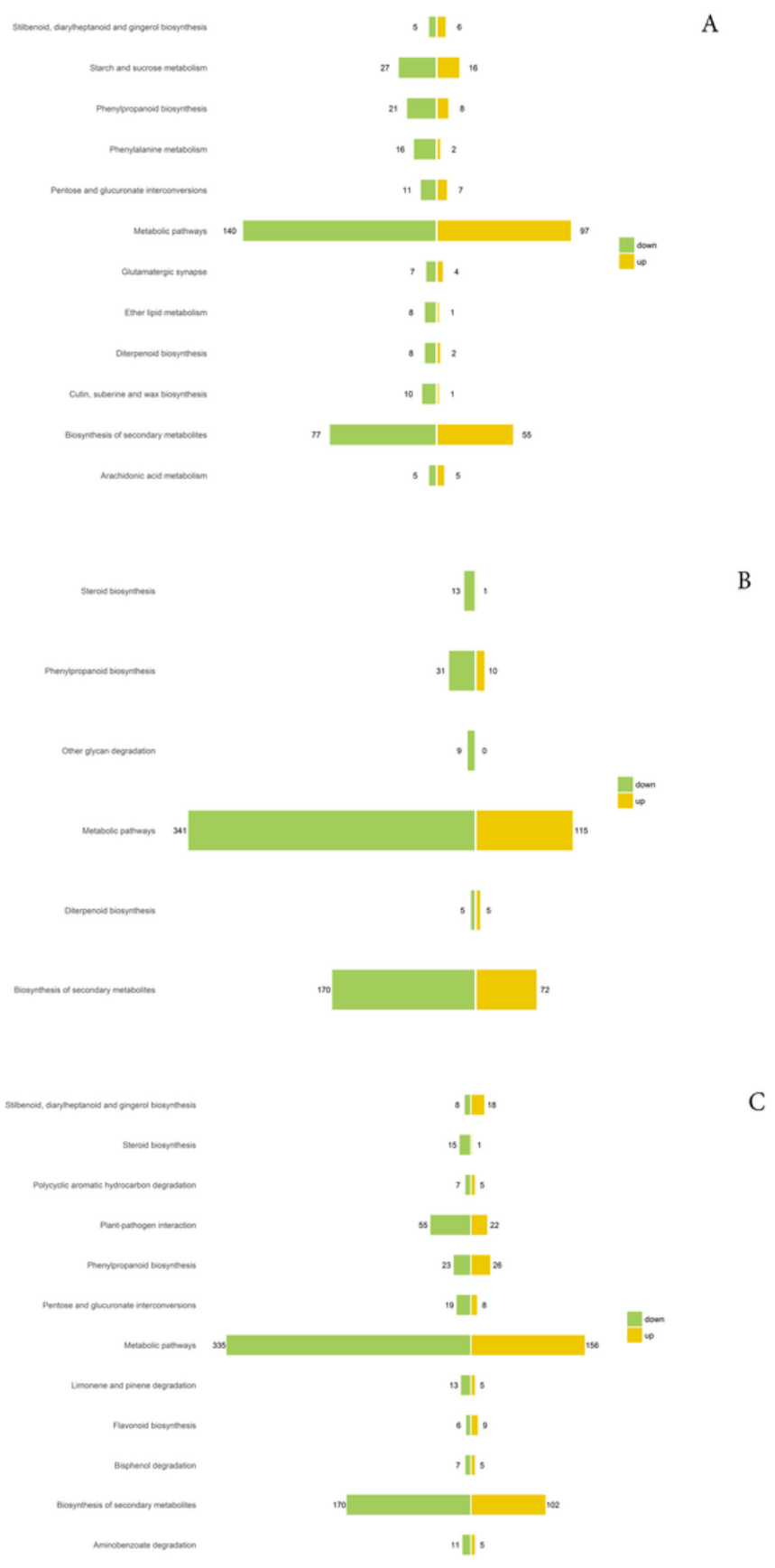

Figure 3

KEGG pathway classification of differentially expressed unigenes of T. hemsleyanum in response to $12 \mathrm{~h}(\mathrm{~A})$, $24 \mathrm{~h}(\mathrm{~B})$, and $48 \mathrm{~h}(\mathrm{C})$ of cold exposure 

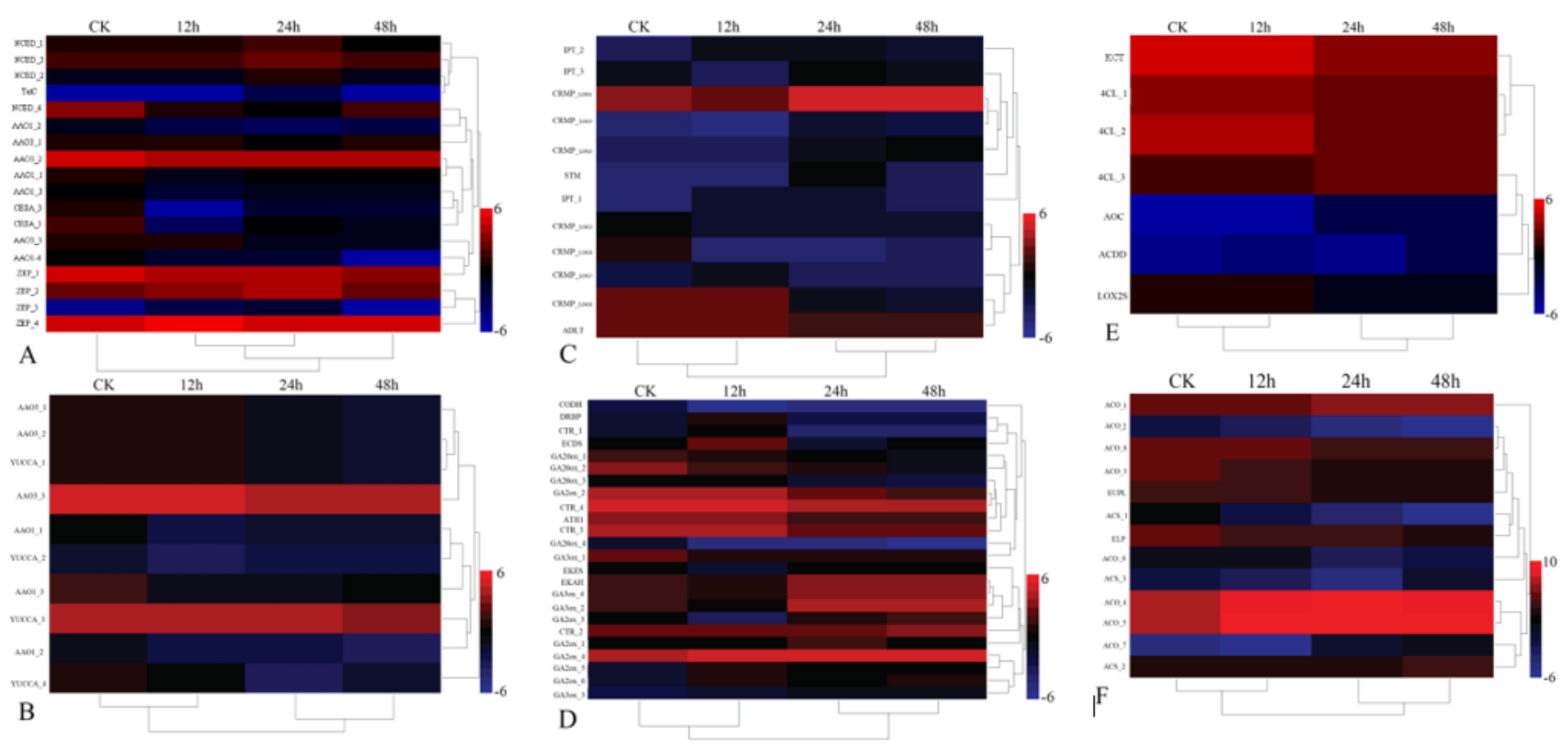

\section{Figure 4}

A heat map indicating expression patterns of homones-related transcripts in $A B A(A)$, Auxin(B), CK(C), GA(D), JA(E), and $E T(F)$ biosynthesis pathways during cold stress obtained from RNA-seq data. A color bar is presented at the bottom right. Data represent the log2 values (RPKM) of the T. hemsleyanum during the CK, $12 \mathrm{~h}, 24 \mathrm{~h}$, and $48 \mathrm{~h}$ stages (from top to bottom) 
A
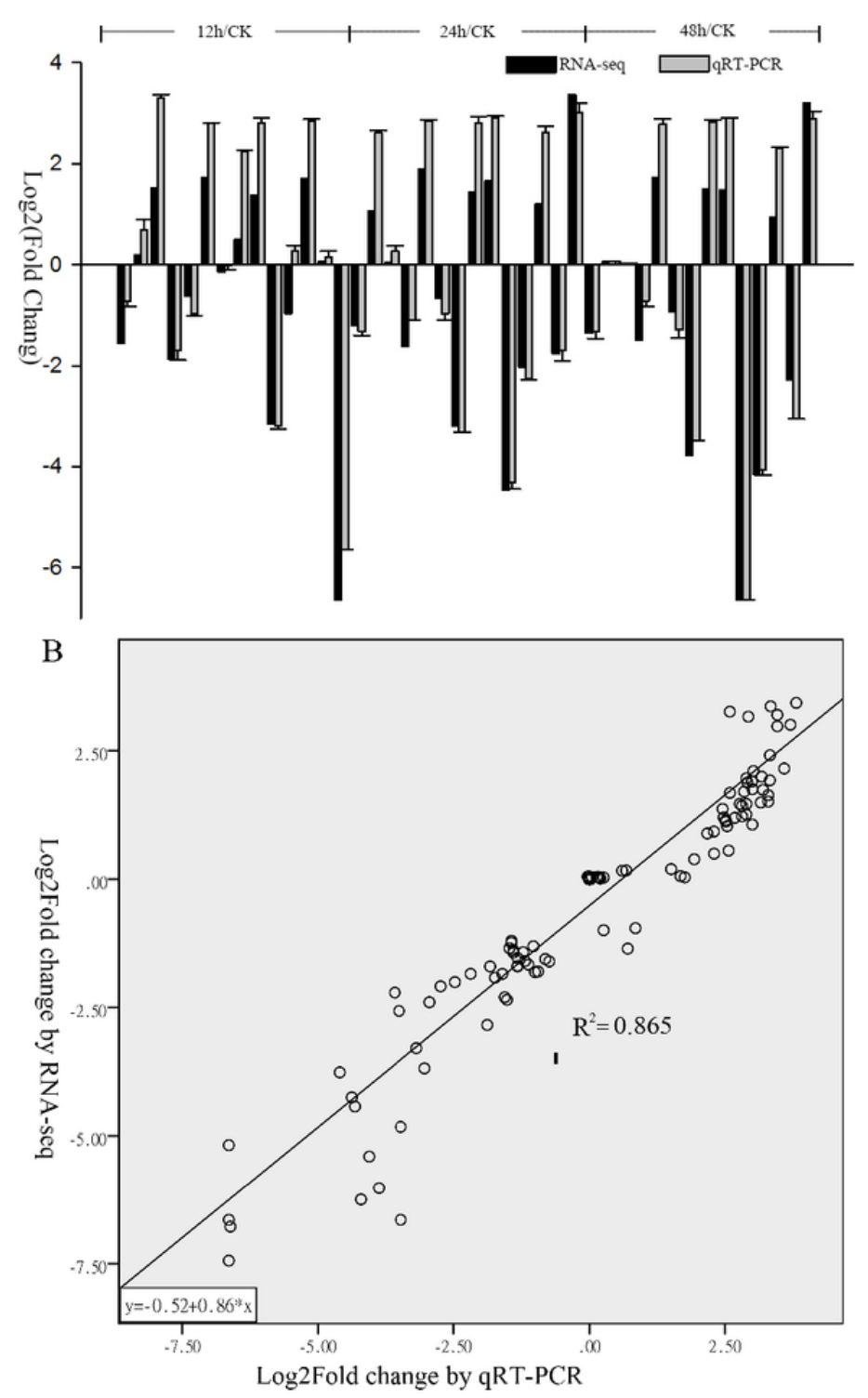

Figure 5

Expression pattern validation (A) and linear dependence relation (B) between the log2 of the key genes expression ratios obtained from RNA-seq and qRT-PCR during cold stress. The transcriptional level of genes was examined by real-time PCR with three biological replicates. RNA-Seq data were highly consistent with qRT-PCR results(R2= 0.865). The candidate genes arranged from left to right as: AAO3_2; NCED_3; ZEP_4; AA01_3; CRMP_LOG1; CRMP_LOG7; CRMP_LOG8; ACO_1; ACO_4; ACS_1; GA20ox_2; GA20x_4; KCT; AOC 

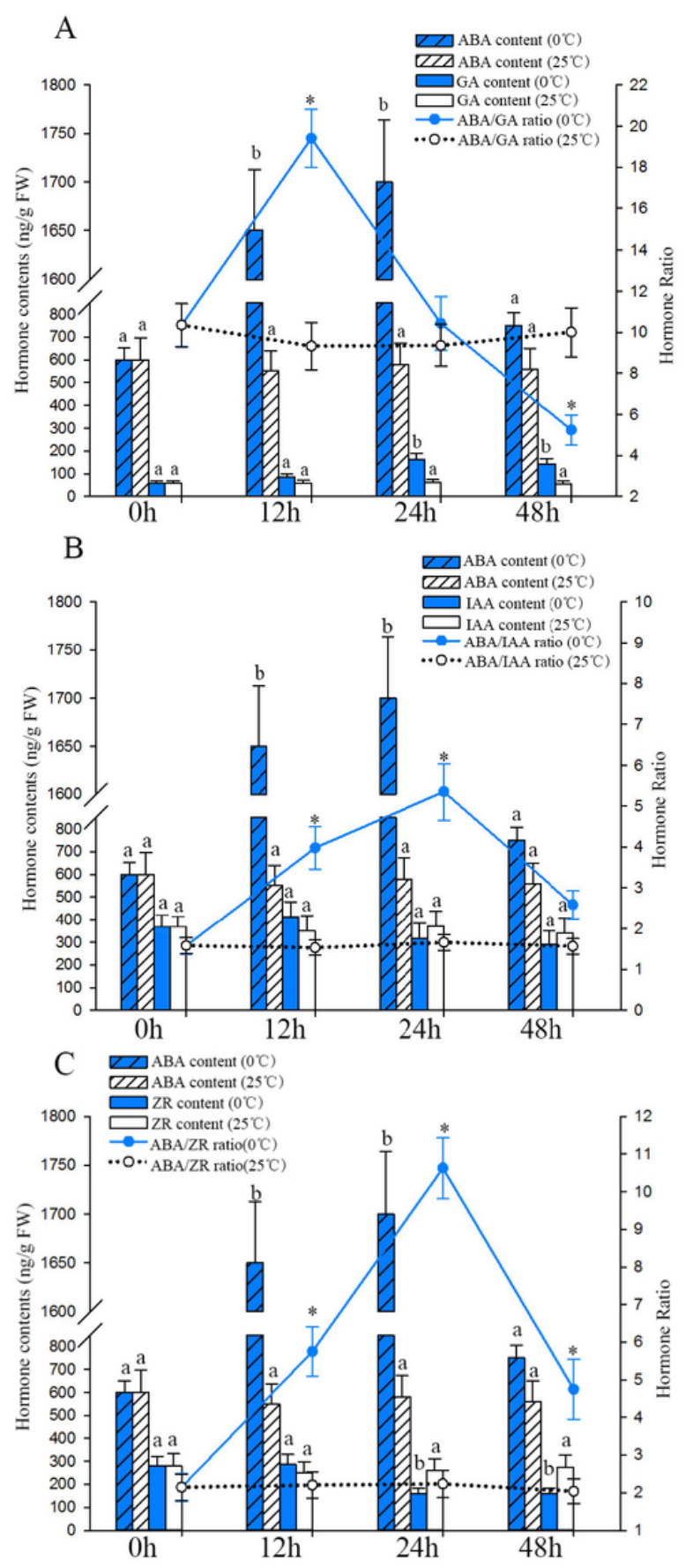

Figure 6

Variation of endogenous phytohormones level and ratio of $A B A / G A(A) ; A B A / I A A(B) ; A B A / Z R(C)$ under $25^{\circ} \mathrm{Cor}$ $0^{\circ} \mathrm{C}$. Values are presented as mean $\pm S D$ of three independent experiments. Bars annotated by the different lowercase letters indicate the significant differences between the samples $(P<0.05)$. Curves annotated by * indicate the significant differences between the treated and control groups $(P<0.05)$ 

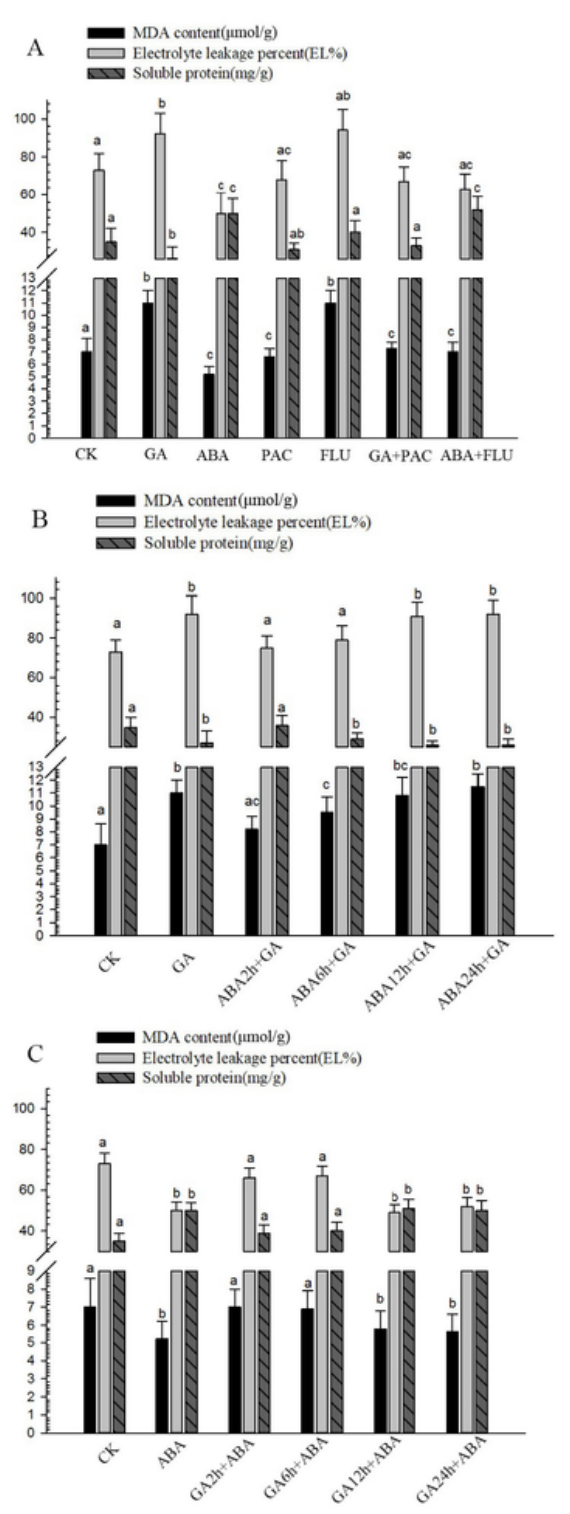

Figure 7

Antagonistic effects of GA and ABA on cold tolerance of T. hemsleyanum. (A) Effect of exogenous GA or ABA used alone or in combination with biosynthesis inhibitors on cold tolerance; $(B)$ Effects of GA on cold tolerance are attenuated by co-application of ABA at 2h, 6h, 12h, and 24h; (C) Effects of ABA on cold tolerance are attenuated by co-application of GA at $2 \mathrm{~h}, 6 \mathrm{~h}, 12 \mathrm{~h}$, and $24 \mathrm{~h}$. Values are presented as mean $\pm S D$ of three independent experiments. Bars annotated by the different lowercase letters indicate the significant differences between the samples $(P<0.05)$.

\section{Supplementary Files}


This is a list of supplementary files associated with this preprint. Click to download.

- Supplementaryfile1ABA2.xIsx

- Supplementaryfile6Cytokinin2.xlsx

- Supplementaryfile4Ethylene2.xIsx

- Supplementaryfile7Brassinosteroid.xlsx

- Supplementaryfile3GA2.xIsx

- Supplementaryfile2AUXIN.xIsx

- Supplementaryfile5JA2.xlsx 